\title{
SOME INEQUALITIES RELATED TO ABEL'S METHOD OF SUMMATION
}

\author{
W. B. PENNINGTON
}

1. It is well known that if

$$
x=e^{-1 / u},
$$

then there exists a constant $\rho$ such that

$$
\limsup _{u \rightarrow \infty}\left|\sum_{n=0}^{\infty} a_{n} x^{n}-\sum_{n \leqq u} a_{n}\right| \leqq \rho \limsup _{n \rightarrow \infty}\left|n a_{n}\right|
$$

for any series $\sum a_{n}$. This inequality is the source of Tauber's 0 -converse of Abel's theorem [Tauber 9]. It is also the source of the following theorem of Vijayaraghavan [10, Theorem 1]:

Theorem 1. Suppose that the series $\sum a_{n} x^{n}$ is convergent for $0<x$ $<1$, to the sum $f(x)$ say, and that, for some fixed real number $\theta, e^{i \theta} f(x)$ $\rightarrow+\infty$ as $x \rightarrow 1-0$. Suppose further that $a_{n}=O(1 / n)$ as $n \rightarrow \infty$. Then $e^{i \theta} \sum_{n \leqq u} a_{n} \rightarrow+\infty$ as $u \rightarrow \infty$.

Theorem 1 may be stated rather less precisely as follows: If the series $\sum a_{n}$ is summable $(A)$ to the sum $s$ with $|s|=\infty$, and if $a_{n}$ $=O(1 / n)$, then $\sum a_{n}=s$. In this form it is seen to be an analogue, for infinite $s$, of Littlewood's well known $O$-Tauberian theorem for Abel summability [Littlewood 8]. Vijayaraghavan showed that the corresponding analogue of the Hardy-Littlewood "one-sided" Tauberian theorem for Abel summability [Hardy and Littlewood 6] is not true. He proved the following theorem [Vijayaraghavan 10, Theorem 3], and showed by an example that it is "best possible."

TheOREм 2. Suppose that the series $\sum a_{n} x^{n}$ is convergent for $0<x<1$, to the sum $f(x)$ say, that $f(x) \rightarrow-\infty$ as $x \rightarrow 1-0$, and that the numbers $a_{n}$ are real and satisfy the inequality

$$
a_{n}>-\frac{K}{n \log \log n}
$$

when $n \geqq 3$. Then

$$
\sum_{n \leqq u} a_{n} \rightarrow-\infty \text { as } u \rightarrow \infty .
$$

It is the object of this paper to obtain an inequality related to

Received by the editors January 7, 1952. 
Theorem 2 in the same way as the inequality (2) is related to Theorem 1.

Recently the inequality (2) and similar ones have received considerable attention $[1 ; 2 ; 3 ; 4 ; 5 ; 7 ; 11]$. Hartman $[7]$ has found the best possible value $\bar{\rho}$ for the constant $\rho$ in $(2)(\bar{\rho}=1.01598 \cdots)$. Agnew $[1 ; 2 ; 2$ contains an account of the previous work on the subject] has shown that if (1) is replaced by $x=e^{-q / u}$, then (2) remains true with $\rho$ depending on $q$, and he has shown that the best possible value $\bar{\rho}(q)$ is least when $q=\log 2(\bar{\rho}(\log 2)=0.96804 \cdots)$.

2. Theorem 2 is clearly a corollary of the theorem:

ThEoREM 3. Suppose that the series $\sum a_{n} x^{n}$ is convergent for $0<x<1$, and that the numbers $a_{n}$ are real. Let

$$
x=\exp \left(-\frac{r}{u(\log u)^{p}}\right),
$$

where $u>0$, and $p$ and $r$ are any fixed real numbers satisfying $p \geqq 1$ and $r>0$. Then

(3) $\quad \liminf _{u \rightarrow \infty}\left\{\sum_{n=0}^{\infty} a_{n} x^{n}-x^{u} \sum_{n \leqq u} a_{n}\right\} \geqq p \liminf _{n \rightarrow \infty} a_{n} n \log \log n$,

and the factor $p$ on the right-hand side is the smallest for which the inequality is true.

The theorem is obviously true if the right-hand side is equal to $-\infty$. We may therefore suppose that

$$
\lim _{n \rightarrow \infty} \inf a_{n} n \log \log n=\alpha>-\infty .
$$

Let $\beta<\alpha$. Then for all but a finite number of the terms $a_{n}$ we shall have

$$
a_{n} n \log \log n>\beta .
$$

It will be sufficient to show that the left side of (3) is greater than $p \beta$.

Since

$$
x^{u}=\exp \left(-\frac{r}{(\log u)^{p}}\right) \rightarrow 1
$$

as $u \rightarrow \infty$, we may change a finite number of the terms $a_{n}$ without changing the value of the left side of (3), and we shall suppose that $a_{0}=a_{1}=a_{2}=0$, and that (4) holds for $n \geqq 3$. 
Then

$$
\begin{aligned}
\sum_{n=0}^{\infty} a_{n} x^{n}-x^{u} \sum_{n \leqq u} a_{n} & =\sum_{n \leqq u} a_{n}\left(x^{n}-x^{u}\right)+\sum_{n>u} a_{n} x^{n} \\
& >\beta\left\{\sum_{8 \leqq n \leqq u} \frac{x^{n}-x^{u}}{n \log \log n}+\sum_{n>u} \frac{x^{n}}{n \log \log n}\right\} \\
& =\beta t(u)
\end{aligned}
$$

say. We have now to show that $t(u) \rightarrow p$ as $u \rightarrow \infty$.

Since $0<x<1$ and $u(\log u)^{p}=-r / \log x$,

$$
t(u) \leqq\left(1-x^{u}\right) \sum_{s \leqq n \leqq u} \frac{1}{n \log \log n}+\frac{1}{\log \log u} \sum_{u \leqq n \leqq u(\log u)^{p}} \frac{1}{n}
$$

$$
+\frac{1}{\log \log u} \sum_{n>-r / \log x} \frac{x^{n}}{n} \text {. }
$$

Now

$$
1-x^{u}=1-\exp \left(-\frac{r}{(\log u)^{p}}\right)=O\left(\frac{1}{(\log u)^{p}}\right)
$$

and

$$
\sum_{3 \leqq n \leqq u} \frac{1}{n \log \log n}<1+\int_{3}^{u} \frac{d v}{v \log \log v}=O\left(\frac{\log u}{\log \log u}\right),
$$

so that the first term on the right side of (6) is

$$
O\left(\frac{1}{(\log u)^{p-1} \log \log u}\right)=O\left(\frac{1}{\log \log u}\right)
$$

since $p \geqq 1$. Since $r>0$,

$$
\begin{aligned}
\sum_{n>-r / \log x} \frac{x^{n}}{n}<1+\int_{-r / \log x}^{\infty} x^{t} \frac{d t}{t} & =1+\int_{1}^{\infty} x^{-v r / \log x} \frac{d v}{v} \\
& =1+\int_{1}^{\infty} e^{-r v v^{-1}} d v<\infty,
\end{aligned}
$$

and so the third term on the right side of (6) is $O(1 / \log \log u)$. It remains to consider the second term. We have

$$
\sum_{1 \leqq n \leqq y} \frac{1}{n}=\log y+O(1)
$$

as $y \rightarrow \infty$, and so 


$$
\begin{aligned}
\sum_{u<n \leqq u(\log u)^{p}} \frac{1}{n} & =\sum_{n \leqq u(\log u)^{p}} \frac{1}{n}-\sum_{n \leqq u} \frac{1}{n} \\
& =\log \left\{u(\log u)^{p}\right\}-\log u+O(1) \\
& =p \log \log u+O(1)
\end{aligned}
$$

as $u \rightarrow \infty$. It follows that the second term is equal to

$$
p+o\left(\frac{1}{\log \log u}\right)
$$

as $u \rightarrow \infty$. Hence, by (6), we have

$$
t(u) \leqq p+o\left(\frac{1}{\log \log u}\right)=p+o(1)
$$

as $u \rightarrow \infty$. On the other hand

$$
\begin{aligned}
t(u) & \geqq \sum_{u<n \leqq u(\log u)^{p} / \log \log u} \frac{x^{n}}{n \log \log n} \\
& \geqq \frac{\exp (-1 / \log \log u)}{\log \log \left\{u(\log u)^{p} / \log \log u\right\}} \sum_{u<n \leqq u(\log u)^{p / \log \log u} \frac{1}{n}}\{p \log \log u+O(\log \log \log u)\} \\
& =\frac{1+O(1 / \log \log u)}{\log \log u+O(\log \log u / \log u)}\left\{p+O\left(\frac{\log \log \log u}{\log \log u}\right)\right. \\
& =p+o(1)
\end{aligned}
$$

as $u \rightarrow \infty$. Thus

$$
t(u) \rightarrow p \text { as } u \rightarrow \infty
$$

and (3) is proved.

The example

$$
a_{n}=\left\{\begin{array}{lr}
0 & (n=0,1,2), \\
-1 / n \log \log n & (n \geqq 3)
\end{array}\right.
$$

proves that the inequality (5) is the best possible, and, consequently, that $p$ is the best possible constant in (3).

This completes the proof of Theorem 3.

If we impose another condition on the series $\sum_{0}^{\infty} a_{n}$, we can remove the factor $x^{u}$ from the left side of (3), and so obtain a closer analogy with (2). 
THEOREM 4. Suppose that the hypotheses of Theorem 3 are fulfilled, and that, in addition, the sum of the series $\sum_{0}^{\infty} a_{n} x^{n}$ is bounded above as $x \rightarrow 1-0$. Then

$$
\liminf _{u \rightarrow \infty}\left\{\sum_{n=0}^{\infty} a_{n} x^{n}-\sum_{n \leqq u} a_{n}\right\} \geqq p \liminf _{n \rightarrow \infty} a_{n} n \log \log n,
$$

and the constant $p$ is the best possible.

Since $\sum_{0}^{\infty} a_{n} x^{n}$ is bounded above,

$$
\begin{aligned}
\sum_{n=0}^{\infty} a_{n} x^{n}-\sum_{n \leqq u} a_{n} & =x^{-u}\left\{\sum_{n=0}^{\infty} a_{n} x^{n}-x^{u} \sum_{n \leqq u} a_{n}\right\}-\left(x^{-u}-1\right) \sum_{n=0}^{\infty} a_{n} x^{n} \\
\geqq & \exp \left(\frac{r}{(\log u)^{p}}\right)\left\{\sum_{n=0}^{\infty} a_{n} x^{n}-x^{u} \sum_{n \leqq u} a_{n}\right\} \\
& +O\left(\frac{1}{(\log u)^{p}}\right)
\end{aligned}
$$

and (8) follows from Theorem 3 since $\exp \left(r /(\log u)^{p}\right) \rightarrow 1$ as $u \rightarrow \infty$.

If we take $a_{n}$ as in (7), we know that

$$
\underset{u \rightarrow \infty}{\liminf }\left\{\sum_{n=0}^{\infty} a_{n} x^{n}-x^{u} \sum_{n \leqq u} a_{n}\right\}=p \liminf _{n \rightarrow \infty} a_{n} n \log \log n,
$$

and

$$
\begin{aligned}
\left\{\sum_{n=0}^{\infty} a_{n} x^{n}-x^{u} \sum_{n \leqq u} a_{n}\right\}-\left\{\sum_{n=0}^{\infty} a_{n} x^{n}-\sum_{n \leqq u} a_{n}\right\} & \\
& =\left(1-x^{u}\right) \sum_{n \leqq u} \frac{1}{n \log \log n} \\
& =O\left(\frac{1}{(\log u)^{p}}\right) o\left(\frac{\log u}{\log \log u}\right) \\
& =O\left(\frac{1}{\log \log u}\right)=o(1)
\end{aligned}
$$

as $u \rightarrow \infty$. Hence

$$
\liminf _{n \rightarrow \infty}\left\{\sum_{n=0}^{\infty} a_{n} x^{n}-\sum_{n \leqq u} a_{n}\right\}=p \liminf _{n \rightarrow \infty} a_{n} n \log \log n,
$$

and since the additional condition imposed in this theorem is obviously satisfied, this completes the proof of the theorem.

We obtain the smallest constant in (3) if we take $p=1$. It is easy 
to see that we cannot take $p<1$ here, for

$$
\begin{aligned}
t(u) \geqq & \sum_{3 \leqq n \leqq u / \log \log u} \frac{x^{n}-x^{u}}{n \log \log n} \\
\geqq & \left\{\exp \left(-\frac{r}{(\log u)^{p} \log \log u}\right)\right. \\
& \left.-\exp \left(-\frac{r}{(\log u)^{p}}\right)\right\} \quad \sum_{3 \leqq n \leqq u / \log \log u} \frac{1}{n \log \log n} \\
= & \left\{\frac{r}{(\log u)^{p}}+o\left(\frac{1}{(\log u)^{p}}\right)\right\}\left\{\frac{\log u}{\log \log u}+o\left(\frac{\log u}{\log \log u}\right)\right\} \\
= & \frac{r}{(\log u)^{p-1} \log \log u}\{1+o(1)\} \rightarrow \infty
\end{aligned}
$$

if $p<1$. However, we can still obtain an inequality if we take

$$
x=\exp (-q \log \log u / u \log u) \quad(q>0) .
$$

THEOREM 5. If

$$
x=\exp (-q \log \log u / u \log u),
$$

where $q$ is a fixed positive number, then

$$
\liminf _{u \rightarrow \infty}\left\{\sum_{n=0}^{\infty} a_{n} x^{n}-x^{u} \sum_{n \leqq u} a_{n}\right\} \geqq(q+1) \liminf _{n \rightarrow \infty} a_{n} n \log \log n .
$$

It is clear that we have to prove that

$$
t(u)=\sum_{3 \leqq n \leqq u} \frac{x^{n}-x^{u}}{n \log \log n}+\sum_{n>u} \frac{x^{n}}{n \log \log n} \rightarrow q+1
$$

as $u \rightarrow \infty$. We have

$$
\begin{aligned}
t(u) \leqq & \left(1-x^{u}\right) \sum_{s \leqq n \leqq u} \frac{1}{n \log \log n}+\frac{1}{\log \log u} \sum_{u<n \leqq u \log u / \log \log u} \frac{1}{n} \\
& +\frac{1}{\log \log u} \sum_{n>-1 / \log x} \frac{x^{n}}{n} \\
= & \left\{\frac{q \log \log u}{\log u}+o\left(\frac{\log \log u}{\log u}\right)\right\}\left\{\frac{\log u}{\log \log u}+o\left(\frac{\log u}{\log \log u}\right)\right\} \\
& +\frac{\log \log u+o(\log \log u)}{\log \log u}+o(1)=q+1+o(1)
\end{aligned}
$$


as $u \rightarrow \infty$. On the other hand

$$
\begin{aligned}
t(u) \geqq & \sum_{3 \leqq n \leqq u / \log \log u} \frac{x^{n}-x^{u}}{n \log \log n}+\sum_{u<n \leqq u \log u /(\log \log u)^{2}} \frac{x^{n}}{n \log \log n} \\
> & \left\{\exp \left(-\frac{q}{\log u}\right)-\exp \left(-\frac{q \log \log u}{\log u}\right)\right\} \\
& \cdot \sum_{3 \leqq n \leqq u / \log \log u} \frac{1}{n \log \log n} \\
& +\frac{\exp (-q / \log \log u)}{\log \log u+o(1)} \sum_{u<n \leqq u \log u /(\log \log u)^{2}} \frac{1}{n} \\
= & q+1+o(1)
\end{aligned}
$$

as $u \rightarrow \infty$. This completes the proof.

It is not difficult to see that if $x=e^{-v}$, where

$$
\begin{gathered}
v=v(u)>0, \quad v=o\left(\frac{\log \log u}{u \log u}\right), \\
1 / v=O(u \log u),
\end{gathered}
$$

then

$$
\liminf _{u \rightarrow \infty}\left\{\sum_{n=0}^{\infty} a_{n} x^{n}-x^{u} \sum_{n \leqq u} a_{n}\right\} \geqq \liminf _{n \rightarrow \infty} a_{n} n \log \log n,
$$

and if $\sum_{0}^{\infty} a_{n} x^{n}$ is bounded above as $x \rightarrow 1-0$,

$$
\underset{u \rightarrow \infty}{\lim \inf }\left\{\sum_{n=0}^{\infty} a_{n} x^{n}-\sum_{n \leqq u} a_{n}\right\} \geqq \liminf _{n \rightarrow \infty} a_{n} n \log \log n .
$$

This is the most precise inequality of this form that we can obtain, and the constant 1 corresponds to Agnew's constant $\bar{\rho}(\log 2)$ $=.96804 \cdots$.

In conclusion we show that the factor $n \log \log n$ in the expression $\lim \inf _{n \rightarrow \infty} a_{n} n \log \log n$ is the smallest that can occur in such onesided inequalities, whatever function $x=x(u)$ we choose.

Suppose that we try to obtain an inequality involving

$$
\liminf _{n \rightarrow \infty} \frac{a_{n} n \log \log n}{\phi(n)}
$$

where $\phi(n) \rightarrow \infty$ as $n \rightarrow \infty$. We shall have to find, if possible, a function $w(u)>0$ such that if $x=e^{-1 / w}$, then 


$$
s(u) \equiv \sum_{3 \leqq n \leqq u} \frac{\phi(n)\left(x^{n}-x^{u}\right)}{n \log \log n}+\sum_{n>u} \frac{\phi(n) x^{n}}{n \log \log n}
$$

is bounded as $u \rightarrow \infty$. We show that this cannot be done.

Let $n_{0}(\geqq 3)$ be such that $\phi(n) \geqq 1$ for $n \geqq n_{0}$. Then

$$
\begin{aligned}
s(u) \geqq & \sum_{n_{0} \leqq n \leqq u / \log \log u} \frac{x^{n}-x^{u}}{n \log \log n} \\
> & \frac{1}{\log \log u}\left\{\exp \left(-\frac{u}{w \log \log u}\right)\right. \\
& \left.-\exp \left(-\frac{u}{w}\right)\right\} \sum_{n_{0} \leqq n \leqq u / \log \log u} \frac{1}{n} \\
= & \frac{(u / w) \log u}{\log \log u}\{1+o(1)\}
\end{aligned}
$$

as $u \rightarrow \infty$. Thus for $s(u)$ to be bounded we must have

$$
w>k \frac{u \log u}{\log \log u}
$$

for some positive constant $k$, as $u \rightarrow \infty$. In particular $w / u$ must tend to infinity with $u$, and so

$$
\begin{aligned}
s(u) & \geqq \sum_{u<n \leqq w} \frac{\phi(n) x^{n}}{n \log \log n}>\frac{e^{-1} \min _{u<\nu \leqq w} \phi(\nu)}{\log \log w} \sum_{u<n \leqq w} \frac{1}{n} \\
& =\frac{e^{-1} F(u) \log (w / u)}{\log \log w}\{1+o(1)\}
\end{aligned}
$$

as $u \rightarrow \infty$, where $F(u)=\min _{u<\nu \leqq w(u)} \phi(\nu) \rightarrow \infty$ as $u \rightarrow \infty$. If $s(u)$ is to be bounded as $u \rightarrow \infty, w(u)$ must certainly satisfy the inequality

$$
\log \frac{w}{u}<K \frac{\log \log w}{F(u)}
$$

for some constant $K$, when $u$ is sufficiently large. We show that the inequalities (9) and (10) are incompatible.

From (10) it follows, in particular, that $\log (w / u)<2^{-1} \log w$, that is to say, $w<u^{2}$, for large $u$. Hence

$$
\log (w / u)<(K / F(u))(\log \log u+\log 2)<(1 / 2) \log \log u
$$

for sufficiently large $u$ since $F(u) \rightarrow \infty$ as $u \rightarrow \infty$. We thus have $w<u(\log u)^{1 / 2}$ as $u \rightarrow \infty$, and this is clearly incompatible with (9). 


\section{REFERENCES}

1. R. P. Agnew, Abel transforms of Tauberian series, Duke Math. J. vol. 12 (1945) pp. 27-36.

2. - Abel transforms and partial sums of Tauberian series, Ann. of Math. (2) vol. 50 (1949) pp. 110-117.

3. H. Hadwiger, Über ein Distanztheorem bei der A-Limitierung, Comment. Math. Helv. vol. 16 (1943-1944) pp. 209-213.

4. - Die Retardierungserscheinung bei Potenzreihen und Ermittlung zweier Konstanten Tauberscher Art, Comment. Math. Helv. vol. 20 (1947) pp. 319-332.

5. - Über eine Konstante Tauberscher Art, Revista Matemática HispanoAmericana (4) vol. 7 (1947) pp. 65-69.

6. G. H. Hardy and J. E. Littlewood, Tauberian theorems concerning power series and Dirichlet's series whose coefficients are positive, Proc. London Math. Soc. (2) vol. 13 (1914) pp. 174-191.

7. P. Hartman, Tauber's theorem and absolute constants, Amer. J. Math. vol. 69 (1947) pp. 599-606.

8. J. E. Littlewood, The converse of Abel's theorem on power series, Proc. London Math. Soc. (2) vol. 9 (1910) pp. 434-448.

9. A. Tauber, Ein Satz aus der Theorie der unendlichen Reihen, Monatshefte für Mathematik und Physik vol. 8 (1897) pp. 273-277.

10. T. Vijayaraghavan, Converse theorems on summability, J. London Math. Soc. vol. 2 (1927) pp. 215-222.

11. A. Wintner, On Tauber's theorem, Comment. Math. Helv. vol. 20 (1947) pp. 216-222.

HARVARD UNIVERSITY 